\title{
Autoimmune Neutropenia in Chronic Liver Disease Patients with Hepatitis C Virus at Suez Canal University Hospital
}

\author{
Nashaat M. Soliman ${ }^{1^{*}}$, Fikry Gobran², Fadia M. Attia ${ }^{2}$, Nashwa R. \\ Elessawy $^{2}$
}

Departments of ${ }^{11}$ nfectious and Endemic Diseases, ${ }^{2}$ Clinical Pathology, Faculty of Medicine, Suez Canal University, Ismailia, Egypt

\begin{abstract}
Background: Chronic hepatitis C ( $\mathrm{CHC}$ ) continues to be a public health problem in Egypt with data suggesting that its incidence may be increasing. Hepatitis $\mathrm{C}$ virus (HCV) infection has been associated with a variety of extra-hepatic manifestations, including autoimmune disorders. Aim: To assess the prevalence of autoimmune neutropenia (AIN) in chronic liver disease (CLD) patients with HCV. Patients and Methods: A case-control design was used to fulfill the study objective. It was conducted on 60 subjects, divided into 2 groups: group 1 ( $30 \mathrm{HCV}$-infected patients with low total leucocytic count [TLC] as a study group) and group 2 (30 HCV-infected patients with normal TLC as a control group). AIN was evaluated by granulocyte immuno-fluorescence test (GIFT) to detect auto-antibodies against neutrophils in HCV-infected patients presented with neutropenia. Results: The mean age of the first group was $49.9 \pm 11.6$ years, while the mean age of the second group was $52.7 \pm 11.3$ years. Male gender was found in $53.3 \%$ of the low TLC group compared to $63.3 \%$ in normal TLC group; Positive GIFT was found in $13.3 \%$ of study group; while none of the control group has positive GIFT. The significant variables among patients with low TLC included low ANC (OR: 5.0, 95\% Cl: 1.5-16.5, low HB (OR: 7.0, 95\% Cl: 1.4-35.5), and low PLT count (OR: 15.5, 95\% Cl: 3.8-63.3). Conclusion: AIN is significantly higher among CHC patients with low TLC compared to normal TLC patients.
\end{abstract}

Keywords: low total leucocytic count, Granulocyte Immuno-fluorescence Test

\section{Introduction}

$\mathrm{HCV}$ infection is a serious health problem affecting over 130 million people worldwide (Ref). Infection with the HCV affects $2-3 \%$ of the world's population $^{(1)}$. Egypt has the highest recorded prevalence of HCV in the world, reaching $14.7 \%$ for $\mathrm{HCV}$-antibody positivity among 15 - to 59-year-old population ${ }^{(2)}$. The viremic population of Egypt was estimated at over 6 million ${ }^{(3)}$. Approximately $85 \%$ of the HCV-infected population develops severe complications, such as $\mathrm{CHC}$, cirrhosis, diabetes, and hepatocellular carcinoma (HCC). About $10-30 \%$ of $\mathrm{CHC}$ patients develop liver cirrhosis. ${ }^{(4,5)} \mathrm{CHC}$ is reported to be the 
major cause of $50-76 \%$ of all liver cancers globally and also the reason for two-third of all cases of liver transplantation. (6) Liver mortality in Egypt reaches 40,000 per year, making $10 \%$ of total mortality, and comes second after heart diseases ${ }^{(7)}$. Primarily, HCV infection appears to be confined to the liver, however, a wide variety of extrahepatic disease manifestations have been reported to be associated with the infection. Extra-hepatic manifestations are mainly due to associated autoimmune disorders. A widely postulated, but controversial, hypothesis is that extra-hepatic disease manifestations are caused by extra-hepatic tropisms of HCV, particularly lymphotropism. The latter is thought to be involved in the production of autoantibodies $^{(8)}$. Persistent HCV infection is responsible for the production of a variety of auto-antibodies including non-organ-specific auto-antibodies and organ-specific auto-antibodies, as a virus-induced autoimmune phenomenon. The diversity of auto-antibodies in the sera of patients with HCV-related CLD has been shown ${ }^{(9,10)}$. Some autoantibodies in $\mathrm{CHC}$ infection have biochemical, histological, or genetic characteristics, while other auto-antibodies may predict the response to anti-viral treatments, concomitant disorders, or prognosis in patients with HCV-related $\mathrm{CLD}^{(11)}$. AIN in adults are a heterogeneous group of diseases with clinical manifestations varying from being asymptomatic to having infectious complications with considerable morbidity and mortality. They are characterized by auto-antibodies directed against neutrophils, resulting in destruction of neutrophils. AIN can be divided into two forms. In primary AIN, neutropenia is usually the sole hematologic abnormality and it is more common in children. Secondary AIN, which is more prevalent in adults, is associated with underlying autoimmune diseases, malignancies, infections, particularly viral, neurological diseases or drug exposure ${ }^{(12)}$. Diagnosis of AIN is based on the presence of neutropenia and demonstration of anti-neutrophil antibodies. The GIFT is a common method that can be used to test for antibodies against neutrophils in both the patient's serum and directly on autologous cells ${ }^{(13)}$.

\section{Patients and Methods}

This case-control study was carried out at Internal Medicine and Clinical Pathology Departments, Suez Canal University Hospitals, Ismailia, Egypt. It included $60 \mathrm{CHC}$ patients. They were classified into two groups; group 1 of $30 \mathrm{CHC}$ patients with leucopenia which considered as the study group and group 2 of $30 \mathrm{CHC}$ patients with normal TLC which considered as the control group. Data were collected from $\mathrm{CHC}$ patients using interview questionnaire. The recorded data included; patients' age, gender, ChildPugh score, and laboratory parameters [hemoglobin (HB), platelets (PLT) count, TLC, absolute neutrophil count (ANC), total and direct bilirubin, Alanine transaminase (ALT), Aspartate transaminase (AST), albumin, total proteins, and prothrombin time (PT)]. GIFT is an indirect immunefluorescence assay for detection of granulocyte auto-antibodies in peripheral blood. Freshly air-dried peripheral 
blood slides were individually wrapped in domestic aluminum foil or paraffin frozen within 2 hours of collection, transported on dry ice and stored at $-25^{\circ} \mathrm{C}$. Frozen slides were reviewed within 2-3 weeks, but were stable for up to 3 months. A peripheral blood sample from normal person was frozen at the same time as each sample and used as a negative control. Two patient slides and the normal control slide were warmed to room temperature and fixed with $1 \%$ para-formaldehyde in phosphate buffered saline (PBS) for 5 min. Fixed neutrophils were incubated with the $1: 2$ dilution of patient serum at $37^{\circ} \mathrm{C}$ for $30 \mathrm{~min}$. Known positive samples were analyzed with each sample batch. Slides were washed with PBS and incubated with fluorescein isothiocyanate (FITC) conjugated to human immunoglobulins $\lg G$ and incubated for further $30 \mathrm{~min}$ at room temperature in the dark. The dilution of the fluorescein conjugated was determined by titration for each batch of antibody (different dilutions of the fluorescein conjugated were applied to peripheral blood control slides incubated with known positive serum, the dilution that gave the best fluorescence was selected). The slides were subsequently rinsed and covered with a PBS-glycerol mountant. The slides were examined microscopically using epifluorescent ultraviolet illumination. A qualitative increase in cytoplasmic fluorescence of patient neutrophils over controls indicated the presence of auto-antibodies ${ }^{(14)}$. Positive and negative controls were run with each batch. The whole slide was reviewed microscopically with the initial result validated by a second review. In cases where the results were not clear, a second individual performed independent analysis of the slides. If the quality of the slide was inadequate (because of sample preparation, storage or transport), these observations were recorded and reported as unsuitable for analysis.

\section{Results}

The age ranged from 24-75 years. The mean age was $49.9 \pm 11.5$ yrs versus $52.7 \pm 11.3$, in the study and the control groups respectively. About 53.3\% were males in the study group compared to $63.3 \%$ in the control group. The groups were matched fro age and gender without statistical significant differences between them ( $p>0.05$ ). There were insignificant differences between the two groups regarding liver function tests and Child-Pugh scoring ( $p>0.05)$ (Table 1). Regarding hematological parameters, there was statistically significant higher mean ANC, HB and PLT count in the control group compared to the study group ( $p<0.01)$ (Table 1). According to GIFT results, the study group had significantly higher incidence of AIN compared to the control group (13.3\% vs. $0.0 \%, p=0.0002$ ) (Table 2 ). The significant variables among patients with low TLC included low ANC, low HB, and low PLT count.

\section{Discussion}

$\mathrm{HCV}$ infection is a common cause of progressive liver disease. In addition, chronic HCV infection has been associ- 
ated with a variety of extra-hepatic manifestations, including autoimmune disorders. HCV may skew the immune response toward the production of auto-antibodies. AIN is a relatively benign disorder characterized by neutrophil specific auto-antibodies, often se- vere neutropenia and a paucity of severe bacterial infections. Antineutrophil antibodies are well recognized causes of neutropenia, producing both quantitative and qualitative defects in neutrophils and increased risk for infection ${ }^{(15)}$.

Table 1: Demographic, clinical and laboratory characteristics of the studied populations

\begin{tabular}{|l|lll|}
\hline Variables & $\begin{array}{c}\text { Study group } \\
(\mathrm{n}=30)\end{array}$ & $\begin{array}{c}\text { Control group } \\
(\mathrm{n}=30)\end{array}$ & p-value \\
\hline Age $(\mathrm{yrs})$ & $49.90 \pm 11.56$ & $52.70 \pm 11.30$ & 0.347 \\
Gender & & & 0.432 \\
$\quad$ Male & $16(53.3 \%)$ & $19(63.3 \%)$ & \\
$\quad$ Female & $14(46.7 \%)$ & $11(36.7 \%)$ & \\
Child-Pugh score & & & 0.094 \\
$\quad$ A & $4(13.3 \%)$ & $0(0.0 \%)$ & \\
B & $17(56.7 \%)$ & $17(56.7 \%)$ & \\
C & $9(30.0 \%)$ & $13(43.3 \%)$ & \\
T. bilirubin $(\mathrm{mg} / \mathrm{dL})$ & $2.69 \pm 2.82$ & $3.65 \pm 4.05$ & 0.096 \\
D. bilirubin $(\mathrm{mg} / \mathrm{dL})$ & $1.25 \pm 0.64$ & $1.68 \pm 0.623$ & 0.589 \\
ALT $(\mathrm{U} / \mathrm{L})$ & $31.43 \pm 19.97$ & $35.60 \pm 24.55$ & 0.375 \\
AST $(\mathrm{U} / \mathrm{L})$ & $56.27 \pm 43.72$ & $61.43 \pm 35.11$ & 0.391 \\
Albumin $(\mathrm{mg} / \mathrm{dL})$ & $2.65 \pm 0.77$ & $2.71 \pm 0.61$ & 0.784 \\
T. proteins $(\mathrm{mg} / \mathrm{dL})$ & $6.18 \pm 1.23$ & $6.65 \pm 0.66$ & 0.135 \\
PT (seconds) & $19.72 \pm 4.99$ & $19.63 \pm 6.41$ & 0.882 \\
TLC (cell/mm $\left./ \mathrm{mm}^{3}\right)$ & $2.44 \pm 0.64$ & $7.11 \pm 2.36$ & $0.001^{* *}$ \\
ANC (cell/mm $\left.{ }^{3}\right)$ & $1.26 \pm 0.22$ & $4.81 \pm 1.99$ & $0.001^{* *}$ \\
HB $($ gm/dL) & $8.64 \pm 1.71$ & $9.83 \pm 1.73$ & $0.017^{*}$ \\
PLT count (cell/mm $\left.{ }^{3}\right)$ & $64.04 \pm 38.68$ & $128.87 \pm 90.43$ & $0.001^{* *}$ \\
\hline
\end{tabular}

Data are presented as Mean $\pm S D$; *Significant $p$-value $\leq 0.05$, **highly significant $p$ value $\leq 0.01$, Alanine transaminase (ALT), Aspartate transaminase (AST), prothrombin time (PT), total leucocytic count (TLC), absolute neutrophil count (ANC), hemoglobin (HB), platelets (PLT).

Autoimmune neutropenia is believed to be triggered by a viral infection (as $\mathrm{HCV}$ ) and, in some individuals, by immunization. When identified in chronic benign neutropenia, antibodies most commonly include immunoglobulin $G$ $(\lg G)$ antibodies against neutrophil glycosylated isoforms. Bone marrow examination typically demonstrates increased cellularity of myeloid ele- ments with an absent or decreased number of mature neutrophils. In some cases, mature forms of neutrophils are seen. This suggests that, in most cases, neutropenia is due to increased consumption of neutrophils in the peripheral circulation and in the tissues that result from phagocytosis of the antibody-coated neutrophils ${ }^{(16)}$. This case-control study was carried out aiming to detect presence of AIN in 
chronic liver disease patients with HCV. When analyzing the results of the laboratory tests, our data showed a statistically significant difference between study and control group regarding ANC, HB and PLT count $(p<0.05)$. In patients with autoimmune neutropenia, hematological evaluation demonstrates a TLC count that is either decreased or within the reference range and a neutrophil count less than

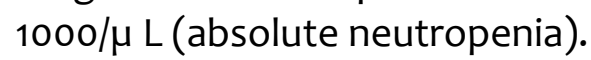

Table 2: Incidence of autoimmune neutropenia (AIN) according to granulocyte immuno-fluorescence test (GIFT)

\begin{tabular}{|c|c|c|c|}
\hline Variables & $\begin{array}{c}\text { Study group } \\
(\mathrm{n}=30)\end{array}$ & $\begin{array}{c}\text { Control group } \\
(\mathrm{n}=30)\end{array}$ & $\mathrm{p}$-value \\
\hline AIN & & & $0.0002^{* *}$ \\
Present & $4(13.3 \%)$ & $0(0.0 \%)$ & \\
Absent & $26(86.7 \%)$ & $30(100.0 \%)$ & \\
\hline
\end{tabular}

**highly significant $p$-value $\leq 0.01$.

Performing sequential $\mathrm{CBC}$ counts with differential to document chronicity is important because most neutropenia in infants resolves with recovery from an acute infection. In individuals with autoimmune neutropenia, the ANC often remains less than $500^{(17,18)}$. Our data revealed that the study group had significantly lower levels of PLT count compared to the control group (64.04 \pm 38.68 versus $128.87 \pm 90.43$, respectively, $\mathrm{p}<0.05)$. Thrombocytopenia is a common hematological toxicity among patients with chronic liver disease, occurring in $15 \%$ up to $70 \%$ of patients with cirrhosis $^{(19)}$

Table 3: Odds ratio and 95\% confident interval of the studied variables among patients with low and normal TLC

\begin{tabular}{|l|lll|}
\hline \multicolumn{1}{|c|}{ Variables } & Odds ratio & $95 \% \mathrm{Cl}$ & -value \\
\hline Age (<40 years) & 2.8 & $0.50-15.7$ & 0.42 \\
Gender (female) & 1.5 & $0.54-4.2$ & 0.43 \\
Total bilirubin & 1.0 & $0.12-8.3$ & 0.70 \\
Direct bilirubin & 0.75 & $0.04-15.0$ & 0.71 \\
ALT & 1.08 & $0.50-2.3$ & 0.50 \\
AST & 0.73 & $0.41-1.3$ & 0.28 \\
Albumin & 1.0 & $0.04-24.5$ & 0.79 \\
Total proteins & 0.86 & $0.044-16.9$ & 0.73 \\
PT & 1.2 & $0.31-4.6$ & 0.79 \\
ANC & 5.0 & $1.5-16.5$ & $0.006^{* *}$ \\
HB & 7.0 & $1.4-35.5$ & $0.0098^{* *}$ \\
PLT count & 15.5 & $3.8-63.3$ & $<0.0001^{* *}$ \\
\hline
\end{tabular}

*Significant $p$-value $\leq 0.05, * *$ highly significant $p$-value $\leq 0.01$, Alanine transaminase (ALT), Aspartate transaminase (AST), prothrombin time (PT), total leucocytic count (TLC), absolute neutrophil count (ANC), hemoglobin (HB), platelets (PLT).

Thrombocytopenia can increase the risk of bleeding associated with inva- sive or surgical procedures, causing delays or cancellations and preventing 
initiation of selected planned therapies $^{(20)}$. The pathophysiology of thrombocytopenia in CLD is complex and multi-factorial. Hypersplenism caused by portal hypertension and subsequent PLT sequestration were thought to be the primary pathogenic mechanisms; however, splenic PLT pooling does not account for thrombocytopenia in all patients; hypersplenism is not always observed in patients with cirrhosis and portal hypertension ${ }^{(21)}$. Some studies in patients with $\mathrm{CHC}$ suggest that HCV may cause direct bone marrow suppression and decreased thrombopoiesis with reduced hepatic production of the thrombopoietin (thrombopoietic growth factor) ${ }^{(22)}$. Other pathogenic mechanisms contribute to the decreased numbers of circulating PLT include increased PLT destruction mediated by immune mechanisms involving anti-platelet auto-antibodies and PLTassociated immune complexes ${ }^{(23,24)}$. Our data reported that about $13.3 \%$ of study group are GIFT positive, while there were no patients in the control group had positive GIFT. There was statistically significance difference between the two groups regarding GIFT. There is no statistically significance between two GIFT results regarding gender and Child-Pugh classifications. So, GIFT results are independent of the degree of liver fibrosis, cirrhosis, and splenomegaly. In Lane et al. ${ }^{(25)}$ study, bone marrow immunofluorescenece test (BMIFT) used to demonstrate auto-antibodies to granulocytes and their precursors on fresh-frozen bone marrow slides. It used to differentiate AIN from other causes of childhood neutropenia. GIFT was used in this study to detect positive anti-neutrophil antibodies from peripheral blood of $\mathrm{HCV}$ patients. Sensitivity for antibody detection varies depending on the test. Indirect GIFT is more sensitive than monoclonal antibody-specific immobilization of granulocyte antigens (MAIGA) in detection of autoimmune neutropenia ${ }^{(26)}$. Antibody specificity is assessed by testing a panel of neutrophils from donors of known human neutrophil antigen (HNA) genotypes. Up to now, the combination of GIFT and granulocyte agglutination (GAT) has been the best means of detection, and this has become the standard approach in most laboratories. GIFT is considered to be the most sensitive of the two methods ${ }^{(27,28)}$. In our study, study group were found to have significantly lower levels of ANC. Positive GIFT was $13.3 \%$ in study group versus $0.0 \%$ in the control group with statistically significant difference. The positive GIFT indicates presence of auto-antibodies against neutrophils in CLD patients with HCV. In Jeffery et al ${ }^{(29)}$ study, patients with HCV infection can develop peripheral blood cell count abnormalities that are commonly attributed to decreased thrombopoietin levels and/ or autoimmune mechanisms. This was emphasized in this study, where cytopenias were occurred due to autoimmune responses in the absence of splenomegaly and/ or antiviral medications, which were excluded from the study. The results of neutrophil antibodies may or may not be positive. Lalezali and colleagues ${ }^{(30)}$ demonstrated antibodies in $98 \%$ of patients with chronic neutropenia, whereas Jonsson and Buchanan ${ }^{(31)}$ demonstrated a posi- 
tive result in only 13 of 28 patients studied. In conclusion, AIN is significantly higher among $\mathrm{CHC}$ patients with low TLC compared to normal TLC patients independent of the degree of liver fibrosis, cirrhosis, and splenomegaly.

\section{References}

1. Alter MJ. Epidemiology of hepatitis $C$ virus infection. World J Gastroenterol. 2007; 13(17): 2436-2441.

2. Benova L, Awad SF, Miller FD, AbuRaddad LJ. Estimation of Hepatitis C Virus Infections Resulting From Vertical Transmission in Egypt. Hepatology. 2015; 61: 834-842.

3. Bruggmann $P$, Berg $T$, Ovrehus $A L$, Moreno C, Brandao-Mello CE, Roudot-Thoraval F. Historical epidemiology of hepatitis C virus (HCV) in selected countries. J Viral Hepat. 2014; 21(Suppl 1): 5-33.

4. Badar S, Khubaib B, Idrees $M$, et al. Association of Hepatitis C Virus with Insulin Resistance: Evidences from Animal Studies and Clinical Studies. Hepat Mon. 2012; 11-15.

5. El-Ezawy H, Hemida M, El-Shiekh $M$. Association of Insulin Resistance, Diabetes Duration and Diabetes Treatment with Risk for Hepatocellular Carcinoma. J Am Sci. 2011; 7(11): 95-101.

6. El-Zanaty F, Way A. Egypt Demographic and Health Survey 2008. Cairo: Ministry of Health, El-Zanaty and Associates, and Macro International, 2009.

7. El-Sayed M. The New National Strategy on Viral Hepatitis. Presentation in a seminar hosted by Al-Ahram Science Clubs, 1 June 2014.

8. Agnello V, De Rosa FG. Extra-hepatic disease manifestations of HCV infec- tion: some current issues. J Hepatol. 2004; 40(2):341-52.

9. Galossi A, Guarisso R, Bellas L, Puoti C. Extra-hepatic manifestations of chronic HCV infection. Journal of Gastrointestinal and Liver Disease. 2007; 16(1): 65-73.

10. Himoto T, Masaki T. Extra-hepatic Manifestations and Auto-antibodies in Patients with Hepatitis C Virus Infection. Clin Dev Immunol. 2012; 2012: 871401.

11. Himoto T, Nishioka M. Autoantibodies in hepatitis $C$ virusrelated chronic liver disease. Hepatitis Monthly. 2008; 8(4):295-303.

12. Mojtaba A, Brian C, Edmund K. Autoimmune neutropenia in adults. Autoimmun Rev. 2009; 9: 62-66.

13. Fromont $P$, Prié $N$, Simon $P$, Cesbron-Gautier A, et al. Granulocyte antibody screening: evaluation of a bead-based assay in comparison with classical methods. Transfusion. 2010; 50(12): 2643-2648.

14. Lalezari P, Jiang AF, Yegen L, Santorineou M. Chronic autoimmune neutropenia due to anti-NA2 antibody. N Engl J Med. 1975; 293(15):744-747.

15. Capsoni F, Sarzi-Puttini P, Zanella A. Primary and secondary autoimmune neutropenia. Arthritis Res Ther. 2005; 7(5): 208-214.

16. Lee $C Y$, Herant M, Heinrich V. Target-specific mechanics of phagocytosis: protrusive neutrophil response to zymosan differs from the uptake of antibody-tagged pathogens. J Cell Sci. 2011; 124(7): 11061114.

17. Inoue S. Autoimmune and Chronic Benign Neutropenia. eMedicine. 2009; 13.

18. Denic S, Showqi S, Klein C, Takala M, Nagelkerke N, Agarwal MM. Prevalence, phenotype and inheritance of benign neutropenia in 
Arabs. BMC Blood Disord. 2009; 27: 9-13.

19. Giannini EG. Review article: thrombocytopenia in chronic liver disease and pharmacologic treatment options. Alim Pharmacol Ther. 2006; 23: 1055-1065.

20. Dieterich DT, Spivak JL. Hematologic disorders associated with hepatitis virus infection and their management. Clin Infect Dis. 2003; 37: 533-541.

21. Adinolfi LE, Giordano MG, Andreana $A$, et al. Hepatic fibrosis plays a central role in the pathogenesis of thrombocytopenia in patients with chronic viral hepatitis. Br J Haematol. 2001; 113: 590595.

22. Wang $\mathrm{C}$, Yao W, Wang A, Chang $\mathrm{T}$, Chou P. Strong association of hepatitis $C$ virus (HCV) infection and thrombocytopenia: implications from a survey of a community with hyperendemic HCV infection. Clin Infect Dis. 2004; 39: 790-796.

23. Doi T, Homma H, Mezawa S, et al. Mechanisms for increment of platelet associated IgG and platelet surface IgG and their implications in immune thrombocytopenia associated with chronic viral liver disease. Hepatol Res. 2002; 24: 23-33.

24. Kajihara $M$, Kato $S$, Okazaki $Y$, et al. A role of autoantibodymediated platelet destruction in thrombocytopenia in patients with cirrhosis. Hepatology. 2003; 37: 1267-1276.

25. Lane SW, Hassell P, Kennedy GA, Fung YL, Williams BA. Characterization of the bone marrow immunofluorescence test in childhood autoimmune neutropenia. Int J Lab Hematol. 2009; 31(5): 567-571.
26. Bux J, Behrens G, Jaeger G, Welte K. Diagnosis and clinical course of autoimmune neutropenia: analysis of 240 cases. Blood. 1998; 91(1):181-186.

27. Bierling $P$, Bux J, Curtis B, et al, ISBT Working Party on Granulocyte Immunobiology. Recommendations of the ISBT Working Party on Granulocyte Immunobiology for leucocyte antibodyscreening in the investigation and prevention of antibody-mediated transfusion-related acute lung injury. Vox San. 2009; 96:266-269.

28. Fromont $\mathrm{P}$, Prié $\mathrm{N}$, Simon $\mathrm{P}$, et al. Granulocyte antibody screening: evaluation of a bead-based assay in comparison with classical methods. Transfusion. 2010; 50(12): 2643-2648.

29. Jeffery $M K$, Bob $G$, Elizabeth $M B$, et al. Bone marrow biopsy in patients with hepatitis $C$ virus infection: spectrum of findings and diagnostic utility. Am J Hematol. 2010; 85:106-110.

30. Lalezari P, Khorshidi M, Petrosova M. Autoimmune neutropenia. J Pediatr. 1986; 109(5):764-769.

31. Jonsson OG, Buchanan GR. Chronic neutropenia during childhood. A 13-year experience in a single institution. Am J Dis Child. 1991; 145(2):232-235. 